\title{
TOLERÂNCIA AO ALUMÍNIO EM PLÂNTULAS DE MILHO
}

\author{
ALUMINUM TOLERANCE IN MAIZE PLANTS
}

\section{Ana Cristina Mazzocato ${ }^{1}$ Paulo Sergio Gomes da Rocha ${ }^{2}$ Maria Jane Cruz de Melo Sereno ${ }^{3}$ Humberto Bohnen ${ }^{4}$ Vanderlise Grongo ${ }^{5}$ José Fernandes Barbosa Neto $^{6}$}

RESUMO

A seleção para tolerância ao alumínio é complicada pela desuniformidade natural dos solos e pela dificuldade de avaliar danos na raiz. Assim sendo, é importante desenvolver métodos eficientes de caracterização da tolerância ao alumínio em condições controladas de ambiente. $O$ presente trabalho teve por objetivo adequar $o$ método de solução mínima para a avaliação da tolerância ao alumínio em genótipos de milho. O trabalho consistiu de dois experimentos. O primeiro foi feito para ajustar o método de avaliação através do emprego de solução mínima, e o segundo, para avaliar um conjunto de 22 genótipos de milho fornecidos pela Empresa Agroeste Sementes Ltda. A avaliação dos genótipos foi realizada com uma solução que continha diferentes concentrações de alumínio e cálcio. As médias para todas as variáveis analisadas mostraram a diferença entre os híbridos e entre as concentrações de alumínio empregadas. As concentrações de $6 m g \ell^{l}$ de alumínio e $40 \mathrm{mg} \ell^{l}$ de cálcio foram consideradas mais eficientes para a discriminação da tolerância ao alumínio, sendo posteriormente empregadas para o trabalho com as linhagens. No segundo experimento, os resultados possibilitaram identificar linhagens com tolerância ao alumínio.

Palavras-chave: alumínio, híbrido, toxidez, solução mínima.

\section{SUMMARY}

Selection for aluminum tolerance is complicated by natural soil desuniformity and difficulty in evaluating root damages. As a consequence, it is important to develop efficient methods of characterization of aluminum tolerance in controlled environment. The present work had the objective of adapting the method of minimum solution for the evaluation of aluminum tolerance in maize genotypes. The work consisted of two experiments. The first try was to adjust the evaluation method through the employment of minimum solution and the second aimed to evaluate a group of 22 maize genotypes supplied by the Agroeste Sementes Ltda. The evaluation was accomplished with a solution of different concentrations of aluminum and calcium. The averages showed differences among the hybrid and the aluminum concentrations. The concentrations of $6 \mathrm{mg}^{1}$ of aluminum and $40 \mathrm{mg} \ell^{l}$ of calcium were considered efficient for the discrimination of aluminum tolerance. This combination was used further to analyze the 22 maize genotypes. In this second experiment, it was possible to identify inbreds with tolerance to aluminum.

Key words: aluminum, hybrid, toxicity, minimal solution.

\section{INTRODUÇÃO}

A maioria dos solos destinados à produção agrícola no Brasil apresenta problemas de acidez e toxidez por alumínio $\left(\mathrm{Al}^{3+}\right)$. O alumínio em níveis tóxicos no solo pode causar efeitos danosos para as plantas, determinando injúrias e retardo do crescimento (MACHADO \& PEREIRA, 1990). A absorção e o acúmulo desse elemento em diferentes partes da planta afetam células e suas organelas em nível morfológico, citogenético e fisiológico, prejudicando seu desenvolvimento, principalmente da parte radicular (KUMAR et al., 1995; WAGATSUMA et al., 1995).

\footnotetext{
${ }^{1}$ Biólogo, Mestre em Fitotecnia. Departamento de Plantas de Lavoura, Faculdade de Agronomia (FA), Universidade Federal do Rio Grande do Sul (UFRGS).

${ }^{2}$ Engenheiro Agrônomo, estudante do Programa de Pós-graduação em Fitotecnia. Departamento de Plantas de Lavoura, FA, UFRGS.

${ }^{3}$ Biólogo, Doutor, Professor Adjunto, Departamento de Plantas de Lavoura, FA, UFRGS.

${ }^{4}$ Engenheiro Agrônomo, PhD., Professor Adjunto, Departamento de Solos (DS), FA, UFRGS.

${ }^{5}$ Engenheiro Agrônomo, estudante do Programa de Pós-graduação em Ciência do Solo. DS, FA, UFRGS.

${ }^{6}$ Engenheiro Agrônomo, PhD., Professor Adjunto, Departamento de Plantas de Lavoura, FA, UFRGS, CP 776, 95501-970, Porto Alegre, RS. jfbn@vortex.ufrgs.br. Autor para correspondência.
} 
A obtenção de altos rendimentos de grãos em milho neste tipo de solo exige a correção da acidez ou o emprego de genótipos tolerantes em níveis considerados tóxicos de alumínio. A seleção para tolerância ao alumínio é complicada pela desuniformidade natural dos solos e pela dificuldade de avaliar danos na raiz, o que determina erros significativos na identificação de genótipos tolerantes em nível de campo. Nesse sentido, é importante desenvolver métodos eficientes de caracterização da tolerância ao alumínio em condições controladas de ambiente. Muitos trabalhos têm empregado solução nutritiva completa para avaliar plântulas tolerantes ao alumínio, demonstrando a presença de variabilidade genética para o caráter em milho (CLARK \& BROWN, 1974; FURLANI \& FURLANI, 1991; DUQUE-VARGAS et $\boldsymbol{a l} .$, 1994). No entanto, a atividade química do alumínio pode ser difícil de avaliar na presença de outros íons. O presente trabalho teve por objetivo adequar o método de solução mínima para a avaliação da tolerância ao alumínio em genótipos de milho, discriminando linhagens de interesse para o melhoramento genético da cultura.

\section{MATERIAL E MÉTODOS}

O trabalho consistiu de dois experimentos, sendo o primeiro para ajustar o método de avaliação através do emprego de solução mínima, e o segundo experimento para avaliar um conjunto de 22 genótipos de milho fornecidos pela Empresa Agroeste Sementes Ltda. Em ambos os experimentos, foram empregados dois híbridos controle, C929 (tolerante ao alumínio) e P32R21 (sensível ao alumínio). Esses híbridos controle foram fornecidos pela Cargill Agrícola S.A. Divisão Sementes, sendo ambos escolhidos com base em prévia experiência de alguns anos de testes em condições naturais de campo. Todos os trabalhos foram realizados no ano de 1999, nos Laboratórios do Departamento de Plantas de Lavoura da Faculdade de Agronomia, da Universidade Federal do Rio Grande do Sul em Porto Alegre (RS).

As sementes de milho foram colocadas para germinar em placas de Petri forradas com papel germinador. As placas de Petri permaneceram em câmara de germinação durante quatro dias a uma temperatura de $25^{\circ} \mathrm{C}$ e fotoperíodo de 12 horas. No segundo experimento, foi adotado o sistema de colocar as sementes em papel germinador enrolado e mantidos de pé sobre um recipiente com água destilada. Após a germinação, as plântulas foram transferidas para uma tela plástica $(2,5 \mathrm{~mm} \mathrm{X}$ $2,5 \mathrm{~mm}$ ) adaptada à tampa de um pote que continha solução tratamento $(8,8 \ell)$. Cada pote recebeu três plântulas e foi considerado como unidade experimental. Os potes foram colocados em banhomaria com iluminação artificial, seguindo um delineamento completamente casualizado com quatro repetições. A água do tanque foi mantida a $25^{\circ} \mathrm{C}$ durante o experimento e as soluções foram aeradas continuamente. No segundo experimento, houve o cuidado de selecionar plântulas com uniformidade de comprimento de radícula.

A solução tratamento continha água destilada e diferentes combinações de $\mathrm{Al}^{3+} \mathrm{e} \mathrm{Ca}^{2+}$. O alumínio foi utilizado na forma de $\mathrm{Al}_{2}\left(\mathrm{SO}_{4}\right)_{3} \cdot 18 \mathrm{H}_{2} \mathrm{O}$ nas concentrações 0,2 e $6 \mathrm{mg}^{-1}$. O cálcio foi adicionado na forma de $\mathrm{CaCl}_{2} \cdot 2 \mathrm{H}_{2} \mathrm{O}$ nas concentrações $20 \mathrm{mg} \ell^{-1}$ e $40 \mathrm{mg} \ell^{-1}$. Esse elemento foi empregado porque é essencial para o crescimento das raízes. Portanto, o seu nível no solo ou em soluções nutritivas é um importante fator a ser considerado na avaliação da tolerância de plantas ao $\mathrm{Al}^{3+}$ (CAMARGO, 1985). O pH de todas soluções foi ajustado com pHmetro na faixa de 4,2 a 4,6, utilizando $\mathrm{NaOH}$ ou $\mathrm{H}_{2} \mathrm{SO}_{4} 1 \mathrm{~N}$.

No momento da transferência das plântulas para a solução tratamento, o comprimento inicial da raiz principal $(\mathrm{cm})$, denominada de primeira leitura (PL), foi medido. Após 48 horas em solução mínima, todos os potes foram trocados para soluções com água destilada e cálcio, permanecendo por mais 48 horas, quando então foi realizada a medida do comprimento final da raiz principal $(\mathrm{cm})$, denominada de segunda leitura (SL). A diferença entre as variáveis PL e SL (SL-PL) foi identificada como DIF $(\mathrm{cm})$ e essa diferença dividida por PL (DIF/PL), tendo como resultante a diferença relativa DIFR. Os dados foram analisados através da análise de variância.

\section{RESULTADOS E DISCUSSÃO}

O desenvolvimento de um método eficiente para caracterizar genótipos com tolerância ao alumínio é o primeiro passo para a realização de estudos de mecanismos genéticos envolvidos na herança desse caráter. A análise da variância indicou que os fatores híbrido e alumínio apresentaram diferenças significativas em todas as variáveis analisadas (Tabela 1), exceto $\mathrm{o}$ fator $\mathrm{Al}^{3+}$ na primeira leitura do comprimento da raiz principal (PL) e o fator híbrido na variável diferença de crescimento relativo (DIFR). A interação entre híbrido e $\mathrm{Ca}^{2+}$ foi significativa para as variáveis segunda leitura do comprimento da raiz principal (SL), diferença entre SL e PL (DIF) e DIFR. Da mesma forma, as interações híbrido $\mathrm{X} \mathrm{Al}^{3+}{\mathrm{e} \mathrm{Al}^{3+} \mathrm{X}}^{3}$ $\mathrm{Ca}^{2+}$ também foram significativas para a variável DIFR.

Ciência Rural, v. 32, n. 1, 2002. 
Tabela 1 - Resumo da análise de variância para os caracteres PL (comprimento inicial da raiz principal - $\mathrm{cm}$ ); SL (comprimento final da raiz principal- $\mathrm{cm}$ ); DIF (diferença entre SL e PL) e DIFR (PL/DIF) medidos em dois híbridos de milho submetidos a diferentes concentrações de $\mathrm{Al}^{3+}$ e $\mathrm{Ca}^{2+}$. Faculdade de Agronomia/UFRGS, Porto Alegre, 1999.

\begin{tabular}{|c|c|c|c|c|c|}
\hline \multirow[b]{2}{*}{ Causas da variação } & \multirow[b]{2}{*}{ GL } & \multicolumn{4}{|c|}{ Quadrados médios } \\
\hline & & PL & SL & DIF & DIFR \\
\hline Híbrido (HIB) & 1 & $74,75^{*}$ & $202,32 *$ & $31,11^{*}$ & 0,49 \\
\hline $\mathrm{Al}^{3+}$ & 2 & 3,74 & $49,42 *$ & $79,16^{*}$ & $4,10 *$ \\
\hline $\mathrm{Ca}^{2+}$ & 1 & 0,03 & 14,54 & 15,79 & 1,43 \\
\hline HIB X Al ${ }^{3+}$ & 2 & 5,49 & 4,04 & 18,18 & $1,63^{*}$ \\
\hline $\mathrm{HIB} \times \mathrm{Ca}^{2+}$ & 1 & 0,09 & $28,00^{*}$ & $31,30^{*}$ & $2,38 *$ \\
\hline $\mathrm{Al}^{3+} \mathrm{X} \mathrm{Ca}^{2+}$ & 2 & 3,17 & 9,11 & 17,39 & $3,23 *$ \\
\hline $\mathrm{HIB} \mathrm{X} \mathrm{Al}^{3+} \mathrm{X} \mathrm{Ca}^{2+}$ & 2 & 3,22 & 1,17 & 7,56 & 1,31 \\
\hline $\mathrm{CV}(\%)$ & & 27,04 & 23,79 & 49,96 & 62,33 \\
\hline
\end{tabular}

* Significativo ao nível de $5 \%$ de probabilidade por F-teste.

O coeficiente de variação oscilou de $23,79 \%$ (SL) a 62,33\% (DIFR), indicando a possibilidade de obter maior precisão na avaliação para tolerância ao $\mathrm{Al}^{3+}$. Essa variação encontrada pode ser explicada por fatores relacionados com a germinação das sementes de milho no presente experimento. Um dos fatores foi o tempo diferente para o início da germinação, no qual o híbrido tolerante (C929) germinava antes do sensível (P32R21), demonstrando desuniformidade para essa variável. Estas observações estão de acordo com as de WAGNER (1999), quando estudou a herança da tolerância ao $\mathrm{Al}^{3+}$ em aveia. Uma medida para reduzir essa desuniformidade poderia ser a utilização de um número maior de sementes para a germinação, com a posterior seleção de plântulas uniformes para a realização dos testes. No entanto, essa seleção deve ser criteriosa, uma vez que plântulas com muitos dias de germinação e com valores elevados para a variável PL já utilizaram as reservas disponíveis no grão, não respondendo ao tratamento com $\mathrm{Al}^{3+}$ e $\mathrm{Ca}^{2+}$. Outro fator a ser considerado foi o pequeno espaço para germinação das sementes em placas de Petri. Foram colocadas cinco sementes de cada híbrido por placa; entretanto, o espaço disponível parece ter dificultado a germinação uniforme e o desenvolvimento das plântulas. Desta forma, uma metodologia que evite o uso de placas de Petri para a germinação seria recomendável.

As médias para todas as variáveis analisadas mostraram a diferença entre os híbridos C929 (tolerante) e P32R21 (sensível) e entre as concentrações de $\mathrm{Al}^{3+}$ (Tabela 2), com exceção da variável DIFR. Esses resultados estão de acordo com
Tabela 2 - Médias para PL (comprimento inicial da raiz principal - $\mathrm{cm}$ ); SL (comprimento final da raiz principal- $\mathrm{cm}$ ); DIF (diferença entre SL e PL) e DIFR (PL/DIF) em dois híbridos de milho submetidos a três concentrações de $\mathrm{Al}^{3+}$. Faculdade de Agronomia/UFRGS, Porto Alegre, 1999.

\begin{tabular}{llcccc}
\hline & & \multicolumn{3}{c}{ Alumínio } \\
\cline { 3 - 5 } Variáveis & Híbridos & $0 \mathrm{mg} \ell^{-1}$ & $2 \mathrm{mg} \ell^{-1}$ & $6 \mathrm{mg} \ell^{-1}$ & Médias \\
\hline \multirow{2}{*}{ PL } & C929 & 6,00 & 7,31 & 8,02 & $7,11 \mathrm{~A}$ \\
& P32R21 & 4,77 & 3,84 & 4,76 & $4,46 \mathrm{~B}$ \\
& Médias & 5,39 & 5,58 & 6,39 & 5,79 \\
SL & C929 & 15,32 & 12,59 & 10,92 & $12,94 \mathrm{~A}$ \\
& P32R21 & 9,92 & 9,25 & 6,57 & $8,58 \mathrm{~B}$ \\
& Médias & $12,62 \mathrm{a}$ & $10,92 \mathrm{ab}$ & $8,75 \mathrm{~b}$ & 10,76 \\
DIF & C929 & 9,32 & 5,29 & 2,90 & $5,84 \mathrm{~A}$ \\
& P32R21 & 5,15 & 5,41 & 1,81 & $4,12 \mathrm{~B}$ \\
& Médias & $7,24 \mathrm{a}$ & $5,35 \mathrm{ab}$ & $2,36 \mathrm{~b}$ & 4,98 \\
DIFR & C929 & 1,61 & 0,83 & 0,36 & 0,93 \\
& P32R21 & 1,25 & 1,75 & 0,44 & 1,15 \\
& Médias & $1,43 \mathrm{a}$ & $1,29 \mathrm{a}$ & $0,40 \mathrm{~b}$ & 1,04 \\
\hline
\end{tabular}

${ }^{1}$ Médias seguidas pela mesma letra, na linha (minúscula) e na coluna (maiúscula), não diferem entre si (Tukey, 5\%).

CANAL \& MIELNICZUK (1983a) e CAMBRAIA \& CAMBRAIA (1995). A variável PL, que foi obtida antes do tratamento com $\mathrm{Al}^{3+}$, representa apenas a diferença entre os genótipos ou variações em decorrência da desuniformidade da germinação, não devendo ser posteriormente analisada.

O híbrido C929 apresentou na média um crescimento superior ao híbrido P32R21, confirmando sua maior tolerância ao $\mathrm{Al}^{3+}$ em níveis elevados na solução tratamento. Entre os sintomas observados pode ser destacada uma redução na parte aérea das plântulas e em suas raízes no híbrido sensível. Esses sintomas são característicos de injúria por toxidez do $\mathrm{Al}^{3+}$ em plantas (FOY, 1974). Diversos autores também têm encontrado diferenças entre genótipos de milho avaliados em solução nutritiva (FURLANI et al., 1986; CAMBRAIA \& CAMBRAIA, 1995).

As raízes principais apresentaram um maior crescimento na ausência de $\mathrm{Al}^{3+}$ na solução (Tabela 2). A concentração de $6 \mathrm{mg} \ell^{-1}$ determinou menores valores para todas as variáveis, com exceção de PL, sendo que a concentração de $2 \mathrm{mg} \ell^{-1}$ apresentou comportamento intermediário. Outros pesquisadores também apontaram a concentração de $6 \mathrm{mg} \ell^{-1}$ como a melhor para a identificação da tolerância ao $\mathrm{Al}^{3+}$ em soluções nutritivas (MARTINS et al., 1999; SIBOV et al., 1999). Por outro lado, outros autores (FURLANI et al., 1986; SAWAZAKI \& FURLANI, 1987; MACHADO \& PEREIRA，1990; FURLANI \& FURLANI，1991) 
utilizaram 4,5mg $\ell^{-1}$ na composição da solução nutritiva, também demonstrando eficiência na seleção de genótipos tolerantes ao $\mathrm{Al}^{3+}$.

$\mathrm{O} \mathrm{Ca}{ }^{2+}$ exerceu uma influência importante na resposta dos híbridos em relação à tolerância ao $\mathrm{Al}^{3+}$, sendo observada uma interação entre o híbrido e essa variável (Tabela 3). De maneira geral, a concentração de $40 \mathrm{mg}^{-1}$ foi a melhor para a identificação de plântulas tolerantes e sensíveis. Por outro lado, CANAL \& MIELNICZUK (1983a) detectaram que a concentração de $40 \mathrm{mg}^{\ell^{-1}}$, mesmo sendo superior a de $0 \mathrm{mg} \ell^{-1}$, não era suficiente para evitar o dano provocado pelo $\mathrm{Al}^{3+}$ em plântulas de milho, já que na presença desse elemento são necessárias quantidades maiores de $\mathrm{Ca}^{2+}$ para o bom desenvolvimento da planta e para a visualização do efeito do $\mathrm{Al}^{3+}$ nos genótipos sensíveis. Os autores também verificaram que o aumento de 20 para $40 \mathrm{mg} \ell^{-1}$ de $\mathrm{Ca}^{2+}$ na solução contribuía para o aumento do comprimento da raiz, tanto em presença como em ausência de $\mathrm{Al}^{3+}$ na solução (CANAL \& MIELNICZUK, 1983b).

A tabela de correlação entre as variáveis PL, SL, DIF e DIFR indicou associações entre todas as variáveis medidas, com exceção da combinação PL X DIF (Tabela 4). Este fato sugere que a melhor variável para avaliação do caráter tolerância ao alumínio é a DIF, uma vez que não esteve associada com a variável PL. É fundamental destacar a importância da medida da raiz principal no início do experimento, possibilitando o cálculo da diferença (DIF). Essas mesmas variáveis, com denominações diferentes, foram utilizadas por SAWAZAKI \&

Tabela 3 - Médias para PL (comprimento inicial da raiz principal - $\mathrm{cm}$ ); SL (comprimento final da raiz principal - $\mathrm{cm}$ ); DIF (diferença entre SL e PL - cm) e DIFR (PL/DIF) em dois híbridos de milho submetidos a duas concentrações de $\mathrm{Ca}^{2+}$. Faculdade de Agronomia/UFRGS, Porto Alegre, 1999.

\begin{tabular}{lcccc}
\hline & \multicolumn{3}{c}{ Cálcio } \\
\cline { 3 - 4 } Variáveis & Híbridos & $20 \mathrm{mg} \ell^{-1}$ & $40 \mathrm{mg} \ell^{-1}$ & \multirow{2}{*}{ Médias } \\
\hline \multirow{2}{*}{ PL } & C929 & $7,08 \mathrm{a}^{1}$ & $7,13 \mathrm{a}$ & $7,11 \mathrm{~A}$ \\
& P32R21 & $4,53 \mathrm{a}$ & $4,38 \mathrm{a}$ & $4,46 \mathrm{~B}$ \\
$\mathrm{SL}$ & $\mathrm{C} 929$ & $13,17 \mathrm{a}$ & $12,71 \mathrm{a}$ & $12,94 \mathrm{~A}$ \\
& P32R21 & $7,18 \mathrm{~b}$ & $9,98 \mathrm{a}$ & $8,58 \mathrm{~B}$ \\
DIF & $\mathrm{C} 929$ & $6,08 \mathrm{a}$ & $5,59 \mathrm{a}$ & $5,84 \mathrm{~A}$ \\
& P32R21 & $2,66 \mathrm{~b}$ & $5,59 \mathrm{a}$ & $4,12 \mathrm{~B}$ \\
DIFR & $\mathrm{C} 929$ & $0,99 \mathrm{a}$ & $0,88 \mathrm{a}$ & 0,93 \\
& P32R21 & $0,73 \mathrm{a}$ & $1,57 \mathrm{a}$ & 1,15 \\
\hline
\end{tabular}

${ }^{1}$ Para cada variável, médias seguidas pela mesma letra, na coluna (maiúscula) e na linha (minúscula), não diferem entre si (Tukey, $5 \%)$.
Tabela 4 - Coeficiente de correlação linear entre as variáveis PL (comprimento inicial da raiz principal - $\mathrm{cm}$ ); SL (comprimento final da raiz principal- $\mathrm{cm}$ ); DIF (diferença entre SL e PL - cm) e DIFR (PL/DIF) em dois híbridos de milho submetidos a diferentes concentrações de $\mathrm{Al}^{3+} \mathrm{e} \mathrm{Ca}^{2+}$. Faculdade de Agronomia/UFRGS, Porto Alegre, 1999.

\begin{tabular}{lccccc}
\hline Variáveis & $\mathrm{n}$ & $\mathrm{PL}$ & $\mathrm{SL}$ & $\mathrm{DIF}$ & DIFR \\
\hline PL & 47 & 1,00 & $0,40^{*}$ & $-0,17$ & $-0,56^{*}$ \\
SL & 47 & & 1,00 & $0,83^{*}$ & $0,43^{*}$ \\
DIF & 47 & & & 1,00 & $0,80^{*}$ \\
DIFR & 47 & & & & 1,00 \\
\hline
\end{tabular}

*Significativo a $5 \%$ de probabilidade.

FURLANI (1987), e os dados de correlação obtidos demonstraram que o crescimento líquido da radícula $(C L R=D I F)$ foi o menos afetado pelo crescimento inicial (CIR) (= PL) e o mais correlacionado com o crescimento final $(\mathrm{CFR}=\mathrm{SL})$, obtido em solução nutritiva com alumínio. Os autores acima citados também consideraram o CLR (= DIF) como a característica mais eficiente para discriminar genótipos de milho quanto ao grau de tolerância ao $\mathrm{Al}^{3+}$. Por outro lado, autores como MAGNAVACA \& BAHIA FILHO (1995) e MARTINS et al. (1999) utilizaram o comprimento relativo da raiz seminal $\left(\right.$ CRRS $=$ DIFR) para avaliar a tolerância ao $\mathrm{Al}^{3+}$, demonstrando ser a única variável que considerava diferenças de vigor de plântula entre os genótipos testados e que apresentava os menores coeficientes de variação para os ensaios.

Com base nos resultados obtidos, 22 genótipos de milho foram testados buscando comprovar a eficiência do método para discriminar plantas quanto à tolerância ao alumínio. A combinação de $6 \mathrm{mg}^{-1}$ e $40 \mathrm{mg} \ell^{-1}$ de $\mathrm{Al}^{3+}$ e $\mathrm{Ca}^{2+}$, respectivamente, foi empregada, sendo a variável DIF medida. Os resultados permitiram identificar dois grupos de linhagens (Figura 1). O primeiro grupo, denominado sensível, composto pelos genótipos L14 até L20, não apresentou diferença estatística em relação ao híbrido sensível P32R21, sendo, entretanto, significativamente diferente do híbrido tolerante C929. Por outro lado, os genótipos constantes do grupo dois (L10, L09 e HS 201) tiveram comportamento oposto, sendo similares ao híbrido C929 e diferentes do P32R21. É importante destacar que, nesta avaliação, a germinação foi realizada em papel germinador e as plântulas com mesmo desenvolvimento radicular foram selecionadas para a realização do tratamento com $\mathrm{Al}^{3+}$ e $\mathrm{Ca}^{2+}$. Esse fato determinou uma redução acentuada do coeficiente de variação (16,7\%), indicando que os procedimentos preconizados em 


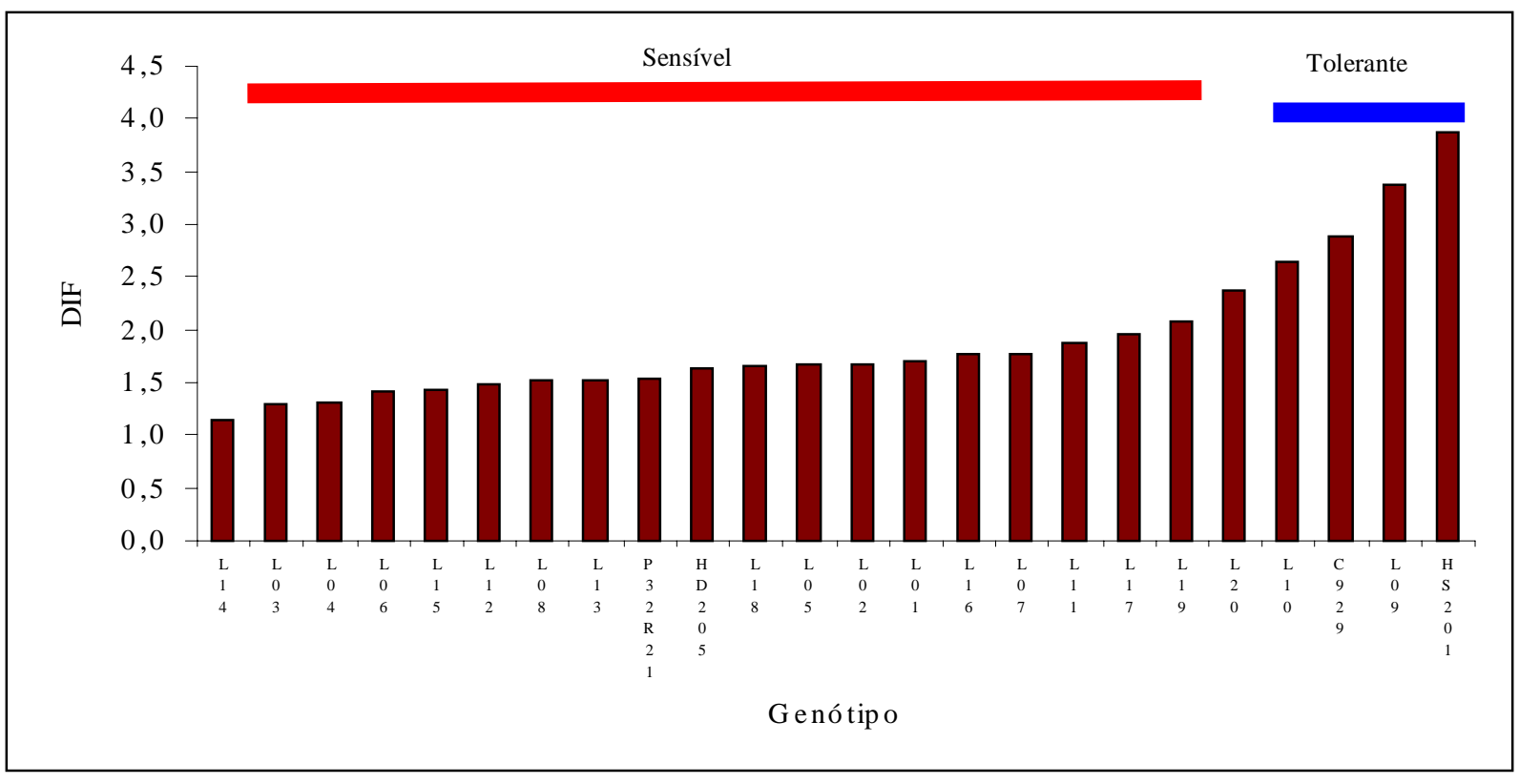

Figura 1 - Comportamento médio da variável DIF (diferença entre SL e PL - cm) de 24 genótipos de milho submetidos ao tratamento com solução mínima contendo 6 e $40 \mathrm{mg}^{\ell^{-1}}$ de $\mathrm{Al}^{3+} \mathrm{e} \mathrm{Ca}^{2+}$, respectivamente. Faculdade de Agronomia/UFRGS, Porto Alegre, 1999.

relação ao experimento anterior foram satisfatórios para diminuir o erro experimental da análise.

\section{CONCLUSÕES}

O método empregado permitiu separar genótipos de milho sensíveis e tolerantes ao alumínio, podendo ser empregado rotineiramente em programas de melhoramento genético.

A melhor combinação das concentrações de $\mathrm{Al}^{3+}$ e $\mathrm{Ca}^{2+}$ para compor a solução tratamento foi de $6 \mathrm{mg} \ell^{-1}$ e de $40 \mathrm{mg} \ell^{-1}$, respectivamente.

Entre as variáveis analisadas no presente trabalho, a variável diferença (DIF) foi a mais eficiente para a detecção da tolerância ao $\mathrm{Al}^{3+}$.

\section{REFERÊNCIAS BIBLIOGRÁFICAS}

CAMARGO, C.E.O. Efeitos de níveis de cálcio combinados com diferentes concentrações de sais na tolerância de trigo á toxicidade de alumínio, em solução nutritiva. Bragantia, v.44, p.659-668, 1985.

CAMBRAIA, J.; CAMBRAIA, M.C. Avaliação de híbridos de milho quanto à tolerância ao alumínio, em solução nutritiva. Ceres, v.42, p.297-307, 1995.

CANAL, I.N.; MIELNICZUK, J. Translocação de cálcio e alumínio em raízes intactas de milho (Zea mays L.). Ciência e Cultura, v.35, p.331-336, 1983a.
CANAL, I.N.; MIELNICZUK, J. Parâmetros de absorção de potássio em milho (Zea mays L.) afetados pela interação alumínio-cálcio. Ciência e Cultura, v.35, p.336-340, 1983b.

CLARK, R.B.; BROWN, J.C. Differential phosphorous uptake by phosphorous stressed maize inbreds. Crop Science, v.14, p.506-508, 1974.

DUQUE-VARGAS, J.; PANDEY, S.; GRANADOS, G., $\boldsymbol{e} \boldsymbol{t}$ al. Inheritance of tolerance to soil acidity in tropical maize. Crop Science, v.34, p.50-54, 1994.

FURLANI, P.R.; FURLANI, A.M.C. Tolerância a alumínio e eficiência a fósforo em milho e arroz: características independentes. Bragantia, v.50, p.331-340, 1991.

FURLANI, P.R.; LIMA, M.; MIRANDA, L.T.; $\boldsymbol{e t}$ al. Avaliação de linhagens, materiais comerciais e duas populações de milho para tolerância a alumínio. Pesquisa Agropecuária Brasileira, v.21, p.655-660, 1986.

KUMAR, P.B.A.N.; DUSHENKOV, V.; MOTTO, H. Phytoextraction: The use of plants to remove heavy metals from soils. Environmental Science Technology, v.29, p.1232-1238, 1995.

MACHADO, E.C.; PEREIRA, A.R. Eficiência de conversão e coeficiente de manutenção da planta inteira, das raízes e da parte aérea em milho e arroz submetidos ao estresse de alumínio. Pesquisa Agropecuária Brasileira, v.25, p.845$855,1990$.

MAGNAVACA, R.; BAHIA FILHO, A.F.C. Seleção de milho para tolerância a alumínio. In: SIMPÓSIO INTERNACIONAL SOBRE ESTRESSE AMBIENTAL, 1, 1992, Belo Horizonte, MG. 
O milho em perspectiva: Anais... Sete Lagoas: EMBRAPA/CNPMS; México, CIMMYT/UNDP, 1995. 449p.

MARTINS, P.R.; PARENTONI, S.N.; LOPES, M.A. et al. Eficiência de índices fenotípicos de comprimento de raiz seminal na avaliação de plantas individuais de milho quanto à tolerância ao alumínio. Pesquisa Agropecuária Brasileira, v.34, p.1897-1904, 1999.

SAWASAKI, E.; FURLANI, P.R. Genética da tolerância ao alumínio em milho cateto. Bragantia, v.46, p.269-278, 1987.
SIBOV, S.T.; GASPAR, M.; SILVA, M.J., et al. Two genes control aluminum tolerance in maize: Genetic and molecular mapping analyses. Genome, v.42, p.475-482, 1999.

WAGATSUMA, T.; ISHIKAWA, S.; OBATA, H., et al. Plasma membrane of younger and outer cells is the primary specific site for aluminum toxicity in roots. Plant and Soil, v.171, p.105-112, 1995.

WAGNER, C.M. Herança genética da tolerância à toxicidade do alumínio em aveia. Porto Alegre, 1999. 69p. Dissertação (Mestrado em Fitotecnia) - Programa de Pós-graduação em Fitotecnia, Faculdade de Agronomia, Universidade Federal do Rio Grande do Sul, Porto Alegre, 1999. 